\title{
MÓDULO DIDÁCTICO PARA EL APRENDIZAJE DE ELECTROMAGNETISMO EN ALUMNOS DEL QUINTO GRADO DE SECUNDARIA DE LA ESPECIALIDAD DE ELECTRICIDAD DE LA I.E. POLITÉCNICO REGIONAL DEL CENTRO - EL TAMBO*
}

\author{
Vilcatoma Sánchez, Amador Godofredoํy Bustamante Altez, Diómedes² \\ Facultad de Pedagogía y Humanidades de la Universidad Nacional del Centro del Perú
}

\begin{abstract}
RESUMEN
La investigación tiene como objetivo determinar la influencia del módulo en el aprendizaje de electromagnetismo en los alumnos del quinto grado de educación secundaria. Es del nivel explicativo, del tipo aplicativo o tecnológico. Se utilizó como método específico el experimental con sus respectivos procedimientos, se aplicó el diseño pre experimental; la técnica de la evaluación, cuyo instrumento fueron las pruebas pedagógicas evaluadas antes y después del experimento. La población estuvo conformada por los estudiantes del quinto grado de secundaria de la especialidad de electricidad y la muestra fue de 19 estudiantes, seleccionada de manera intencional, no probabilística, porque se eligió la sección ya establecida previo a la aplicación de la variable independiente. Luego de la prueba postexperimental se demostró que el módulo educativo influye positivamente en el aprendizaje de electromagnetismo en los alumnos del quinto grado de educación secundaria, además esta influencia se demuestra a nivel del grupo experimental como también al evaluar después de 20 días. La aceptación de la hipótesis fue mediante el modelo estadístico de la t de student para datos dependientes e independientes con $=0,05$
\end{abstract}

Palabras clave: módulo, aprendizaje, electromagentismo.

\begin{abstract}
The investigation aims to determine the influence of the module on learning of electromagnetism in the fifth grade students of secondary education. It is the standard explanation, the rate applied or technological. Specific method was used as the pilot with their own procedures, was applied pre experimental design, technology assessment, whose pedagogical tool tests were evaluated before and after the experiment. The population was made up of fifth grade students of secondary specialty electricity and the sample was 19 students, chosen deliberately, not probabilistic, was chosen because the section already in place prior to the implementation of the independent variable. After the post-experimental test showed that the educational module has a positive impact on learning of electromagnetism in the fifth grade students of secondary education, this influence was also shown at the experimental group as well as to evaluate after 20 days. Accepting the hypothesis was using the statistical model of the $t$ student data dependent and independent for $=0,05$.
\end{abstract}

Key words: module, learning, electromagentismo.

* Este trabajo de invetigación fue recibido el 20/03/2007, retornado para su revisión el 22/09/2008 y aprobado para su publicación el 27/02/2009.

1. Email: evilcatoma58@hotmail.com

2. Email: diomedes@hotmail.com 


\section{INTRODUCCIÓN}

El motivo fundamental que nos impulsó a realizar la presente investigación, es el aspecto metodológico que existe dentro del área de la enseñanza de la Física; donde predomina la aplicación de métodos, técnicas y procedimientos que no permiten la participación activa, consciente y socializada de los alumnos y maestros, así como también la aplicación inadecuada de las técnicas, con lo cual los alumnos son meramente repetitivos, pasivos memorísticos lo que influye al bajo rendimiento escolar, poca comunicatividad, creatividad e ingeniosidad, para lo cual se ha formulado la siguiente interrogante: ¿de qué manera influye el módulo didáctico en el aprendizaje de electromagnetismo en los alumnos del quinto grado de educación secundaria de la Institución Educativa Politécnico Regional del Centro de Huancayo?

La hipótesis que guió la investigación fue: el módulo educativo influye positivamente en el aprendizaje de electromagnetismo en los alumnos del quinto grado de educación secundaria de la institución educativa Politécnico Regional del Centro del Perú de Huancayo.

En el proceso de la investigación se ha utilizado el método experimental con un diseño preexperimental, que fue base para la investigación.

El trabajo de investigación para su comprensión y desarrollo se ha estructurado de la siguiente manera: el primer capítulo describe el planteamiento del estudio; el segundo capítulo se refiere al marco teórico, donde encontramos los antecedentes del estudio y los fundamentos teóricos, tanto en el aspecto metodológico y físico. En el tercer capítulo abordamos el aspecto metodológico y en el cuarto capítulo desarrollamos el análisis e interpretación de los resultados obtenidos en el proceso de investigación. Luego se redactaron las conclusiones, sugerencias, también se incluyen los anexos en donde presentamos los instrumentos empleados.

El objetivo general fue determinar la influencia del módulo didáctico en el aprendizaje de electromagnetismo en los alumnos del quinto grado de educación secundaria de la institución educativa Politécnico Regional del Centro de Huancayo.

\section{MATERIAL Y MÉTODOS}

En el presente trabajo se utilizó el tipo de investigación tecnológico o aplicado; el cual se caracteriza por su utilidad al aplicar ciertos conocimientos teóricos a una determinada situación concreta y a las consecuencias prácticas que de ella se derivan.

Durante la investigación se hizo uso del método científico, partiendo de una hipótesis determinada. En la ejecución de la investigación se tuvo en cuenta el método experimental porque presupone procedimientos del más riguroso control.

En el trabajo de investigación se aplicó el diseño preexperimental, utilizando un grupo experimental cuyo diagrama es el siguiente:

\section{G.E: $\quad 01 \quad X \quad 02 \quad 03$}

- G.E.: Grupo experimental.

- 01: Aplicación del pretest (prueba de entrada).

- X: Aplicación de la variable independiente o experimental.

- 02: Aplicación del posttest (prueba de salida).

- 03: Aplicación de posttest después de 20 días.

La población del trabajo de investigación estuvo conformada por los alumnos del quinto grado de educación secundaria, turno noche de la institución educativa Politécnico Regional del Centro de Huancayo y la muestra estuvo constituida por 19 alumnos que corresponden al quinto grado de educación secundaria, que fue seleccionada de manera intencional, no probabilística. Entre las técnicas utilizadas se tienen el fichaje y la evaluación, en tanto que, como instrumentos de recolección de datos, se utilizaron fichas bibliográficas y fichas de resumen para recolectar las informaciones más importantes y las pruebas pedagógicas de entrada y de salida que sirvieron para evaluar los contenidos conceptuales y procedimentales.

Se obtuvieron los datos de la investigación utilizando los siguientes procedimientos:

- Se coordinó con el director del plantel para elegir la muestra.

- La validez y confiabilidad de la prueba de entrada y salida se realizó la segunda y tercera semana del mes de julio del 2006.

- Se sensibilizó a los alumnos mediante el diálogo 
acerca de la importancia de conocer y aprender el tema de electromagnetismo.

- Los alumnos de ambas secciones se sometieron a una prueba de entrada (pretest). Esta prueba tuvo una duración de 50 minutos y se realizó el 17 de agosto del 2006.

- Luego, se llevó a cabo el experimento en el grupo experimental, teniendo una duración de un mes, aplicando la variable independiente.

Finalmente, se aplicó el postest (prueba de salida), en dos etapas esto con el propósito de determinar la eficacia de la aplicación del módulo.

\section{RESULTADOS}

Análisis descriptivo de las medidas de tendencia central.

Cuadro 1. Resumen de las medidas estadísticas de la prueba de entrada y salida.

\begin{tabular}{ccc}
\hline & $\begin{array}{c}\text { Prueba } \\
\text { de salida }\end{array}$ & $\begin{array}{c}\text { Prueba } \\
\text { de entrada }\end{array}$ \\
\hline Media & 12,84 & 9,84 \\
ediana & 13 & 10 \\
Moda & 12 & 11 \\
Desv. típ. & 1,119 & 1,302 \\
Varianza & 1,251 & 1,696 \\
\hline \multirow{2}{*}{ Coefic. Variac. } & $8,7 \%$ & $13,23 \%$ \\
& Homogéneo & Homogéneo \\
\hline
\end{tabular}

- En cuanto a la media aritmética, se obtiene el valor de 12,84 en la prueba de salida, mayor a comparación de la prueba de entrada que es de 9,84, mostrando una diferencia de 3 puntos.

- En cuanto a la mediana en la prueba de salida es que el $50 \%$ de los alumnos obtienen una nota superior e inferior a 13, mientras que en la prueba de entrada las notas son superiores e inferiores de 10.

- La nota de mayor frecuencia en la prueba de salida es 12, mientras que en la prueba de entrada es 11.

- Se presenta menor dispersión en la prueba de salida y una dispersión relativa mayor en la prueba de entrada.

- En ambas evaluaciones los puntajes obtenidos son homogéneos en cuanto a sus varianzas.

Docimasia de hipótesis. t de student

Hipótesis de trabajo.
H0: No existe diferencia significativa en el puntaje promedio obtenido en el posttest después de la aplicación del módulo didáctico para el aprendizaje de electromagnetismo en los alumnos del quinto grado de educación secundaria de la especialidad de electricidad, en comparación con el pretest con un $=0,05$.

$$
\text { Ho: } \mu_{1}=\mu_{2}
$$

H1: El puntaje promedio obtenido en el posttest después de la aplicación del módlo didáctico para el aprendizaje de electromagnetismo en los alumnos del quinto grado de educación secundaria de la especialidad de electricidad a comparación con el pretest con un $\alpha=0,05$.

$$
H a: \mu_{1}>\mu_{2}
$$

Prueba estadística : : "t de student".

Nivel de significación $\quad: \alpha=0,05$.

Grados de libertad $\quad: \mathrm{gl}=\mathrm{n} 1-1 \mathrm{gl}=18$

Punto crítico $\quad: \mathrm{t}_{0,05}=1,7291$

Decisión Si $t_{c}>t_{0,05}$ se rechaza la $H_{0}$.

Si $\mathrm{t}_{\mathrm{c}}<\mathrm{t}_{0,05}$ se acepta la $\mathrm{H}_{\mathrm{o}}$

\begin{tabular}{lllc}
\hline & Media & $\mathrm{N}$ & Desviac. típ \\
\hline Prueba de entrada & 9,84 & 19 & 1,302 \\
Prueba de salida & 12,84 & 19 & 1,119 \\
\hline
\end{tabular}

La razón t. $\quad t_{c}=9,29$.

Decisión. Se rechaza la hipótesis nula y se acepta la hipótesis alterna, o sea que: de la aplicación del módulo didáctico influye positivamente para el aprendizaje de electromagnetismo en los alumnos del quinto grado de educación secundaria de la especialidad de electricidad, con $\alpha=0,05, \mathrm{gl}=18$, en donde la $\mathrm{t}_{\mathrm{c}}=$ 9,29 es mayor que la $t_{t}=1,7291$.

\section{DISCUSIÓN}

La concepción constructivista de aprendizaje adoptada por el planteamiento curricular, rompe con la tradicional confrontación entre métodos de enseñanza centradas en el alumno; activos, abiertos, progresivos y los métodos de enseñanza centrados en el profesor; 
receptivos, cerrados, expositivos y tradicionales. (J. Escaño citado por Cox, 2002 p.14).

Pero aún en la actualidad hay un gran número de salas de clases, en donde el libro para el alumno, es el único material importante que se utiliza en el proceso de enseñanza-aprendizaje. Poco a poco se han ido implantando modificaciones en el sistema de enseñanza-aprendizaje de la física y se han incorporado el uso de nuevos materiales, en donde los materiales manipulativos han permitido una adquisición del aprendizaje a través de la acción sensorio-motriz.

Para Vigotsky "lo que crea la zona de desarrollo próximo es un rasgo esencial de aprendizaje; es decir, el aprendizaje despierta una serie de procesos evolutivos internos capaces de operar cuando el niño está en interacción con las personas de su entorno y en cooperación con algún semejante". (Vigotsky, 1988, p.138). Sin embargo, no se considera eficiente a todo trabajo en cooperación con alguien que sabe más; la idea es que se trabaje con alguien que sabe más sobre un concepto que el niño desarrollará e internalizará en un futuro próximo. Debe quedar claro que la noción de ZDP hace referencia a trabajar sobre un nivel evolutivo por desarrollarse, no sobre lo ya desarrollado, es decir que no es una mera práctica.

El aprendizaje no es desarrollo pero "el aprendizaje organizado se convierte en desarrollo mental y pone en marcha una serie de procesos evolutivos que no podrían darse nunca al margen del aprendizaje" (Vigotsky, 1988, p.139).

Esta aseveración es confirmada en la presente investigación al concluir que efectivamente el módulo didáctico influye positivamente en el aprendizaje del tema de electromagnetismo en los alumnos del quinto grado de secundaria, tal como lo corrobora los trabajos realizados por Díaz y Álvarez (1993); Pomalaza (1994) y de Valenzuela y Yabar (1991).

Así, mientras más variados y significativos son para el estudiante los contactos con el mundo que le proporcione la institución por medio de las actividades, mayor serán sus bases para el desarrollo del pensamiento lógico y mayor su sensibilidad para el aprendizaje, puesto que los materiales educativos y en este caso el módulo son recursos didácticos que permiten y facilitan la enseñanza y el aprendizaje, produciendo los cambios deseados en los estudiantes.

En lo que respecta al cambio en la disposición por parte de la muestra, ésta fue en forma positiva, se evidenció un cambio sustancial, ya sea en su comportamiento del proceso, como de igual forma en las respuestas entregadas.

Lo antes mencionado concuerda con lo expresado por Dweck citado por Katz, 2000, quien señala que "para adquirir o fortalecer una disposición en particular, un niño debe tener la oportunidad de expresar la disposición en su comportamiento. Cuando ocurren manifestaciones de las disposiciones, éstas pueden fortalecerse cuando el niño observa su efectividad, en las respuestas.

\section{CONCLUSIONES}

- El puntaje promedio obtenido es mayor en el postest después de la aplicación del módulo educativo en el aprendizaje de electromagnetismo en los alumnos del quinto grado de educación secundaria de la institución educativa Politécnico Regional del Centro a comparación con el pretest, tal como se demuestra estadísticamente a un nivel de significancia del 0,05.

- La diferencia entre el puntaje promedio obtenido en el postest y la prueba recurrente aplicada después de 20 días es significativamente positiva, es decir el módulo es eficaz para el aprendizaje de electromagnetismo, en los alumnos del quinto grado de educación secundaria de la institución educativa Politécnico Regional del Centro.

\section{LITERATURA CITADA}

Ary, D. 1994. Introducción a la investigación pedagógica, Segunda edición, Edit. Alhambra.

Avila, A. R. 2001. Estadística elemental. Edit. Estudios y Ediciones. Lima - Perú.

Baquero, R. 1996. Vigotsky y el aprendizaje escolar. Buenos Aires: Aique. 
Córdova, Z. M. 1996. Estadística descriptiva e inferencial. Segunda edición, Editorial Moshera.

Cox, A; Labbé E; Yañez P. 2002. Enseñanza y aprendizaje de las figuras geométricas, Tesis para optar al título de profesor en educación básica, Universidad Católica de Chile.

Díaz, B. F. y Hernández. 1998. Estrategias docentes para un aprendizaje significativo. Mc.Graw Hill. México.

Díaz, y Álvarez. 1993. El uso de módulos didácticos en la enseñanza-aprendizaje del sistema excretor en el 4to. grado de educación secundaria en el colegio Chinchaysuyo de Sapallanga.

Dubrovsky, S. 2000. Vigotsky. Su proyección en el pensamiento actual. Buenos Aires: Ediciones Novedades Educativas.

Etollberg, R. 1999. Física. Fundamentos y frontera. Ed. Faith Fitch Hill.

Flores, O. R. 1994. Hacia una pedagogía del conocimiento. Edit. Mc. Graw Hill. Colombia.

Gallimore, R. y Tharp R. 1999. Concepción educativa de la sociedad. Edic. Koper. España. 1999.

Gran, M.F. 2001. Elementos de física general y experimental. Tomos I y II. Ed. Minerva Books.

Hernández, S. R. 1994. Metodología de la investigación. Edit. McGraw-Hill. Colombia. 1994.

Katz, L. 2000. Otra perspectiva sobre lo que los niños deben estar aprendiendo; Artículo publicado en: http://www.ericdigests.org/2000-4/.htm visitada el 28 octubre de 2005.

Konmikova y otros. 1996. Metodología de la labor educativa. Edit. Grijalbo, México.
Newman, D., Griffin, P., Cole, M. 1991. La zona de construcción del conocimiento. Madrid: Morata.

Orozco, G. 1991. Educación para la recepción. Instituto Latinoamericano de Comunicación Educativa, México.

Pomalaza. 1994. Diseñar módulos didácticos de gráficos del movimiento mecánico para utilizar como técnica auxiliar en el aprendizaje de la física la cual fue aplicada en el centro educativo "José Carlos Mariategui" de San Pedro de Sanos.

Sánchez, C. H. y Reyes M. C. 1996. Metodología y diseños en la investigación científica. Primera edición: marzo - Perú.

Soto, M. V. 2003. Organizadores del conocimiento. Edit. Razuwillca. Huancayo-Perú.

Spiegel, M. R. 1980. Estadística. Libros Mc Graw Hill - México.

Valenzuela y Yabar, M. 1991. Aplicación de guías de práctica para la enseñanza de algunas medidas de tendencia central en alumnos del 3er grado de educación secundaria del C.E. "Mariscal Castilla" El Tambo - Huancayo.

Véliz, C. V. 1998. Estadística: aplicaciones. Copyright. Lima - Perú.

Vigotsky, L. 1988. El desarrollo de los procesos psicológicos superiores. Editorial Crítica, Grupo Editorial Grijalbo; México.

Vigotsky, L. 1995. Pensamiento y lenguaje. Buenos Aires: Ediciones Fausto. 